\title{
New Lanark ou les origines écossaises d'un village industriel « modèle »
}

New Lanark: a Model Industrial Village in the Scottish Context

\section{Ophélie Siméon}

\section{(2) OpenEdition \\ 12 Journals}

\section{Édition électronique}

URL : https://journals.openedition.org/etudesecossaises/730

DOI : 10.4000/etudesecossaises.730

ISSN : 1969-6337

\section{Éditeur}

UGA Éditions/Université Grenoble Alpes

\section{Édition imprimée}

Date de publication : 15 avril 2012

Pagination : 183-200

ISBN : 978-2-84310-223-3

ISSN : $1240-1439$

\section{Référence électronique}

Ophélie Siméon, « New Lanark ou les origines écossaises d'un village industriel « modèle » », Études écossaises [En ligne], 15 | 2012, mis en ligne le 15 avril 2013, consulté le 11 avril 2023. URL : http:// journals.openedition.org/etudesecossaises/730 ; DOI : https://doi.org/10.4000/etudesecossaises. 730 


\section{New Lanark ou les origines écossaises d'un village industriel « modèle »}

Contrairement aux représentations courantes, l'usine n'est pas uniquement une réalité urbaine. Entre 1770 et 1830, de nombreuses mines et filatures ont été implantées en milieu rural, logique mue par la nécessité de s'établir à proximité des sources de matière première et d'énergie. Fonctionnant souvent à l'énergie hydraulique, de nombreuses usines textiles sont ainsi bâties en amont de cours d'eau puissants. La première révolution industrielle voit donc naître une institution originale, à mi-chemin entre la fabrique et la grande usine urbaine : le village ouvrier.

Cette étude porte sur le plus célèbre d'entre eux, le village ouvrier « modèle » de New Lanark. Fondée en 1785 sur les rives de la Clyde par David Dale, banquier et négociant de Glasgow, la filature acquiert rapidement une réputation d'entreprise éclairée. Comme d'autres industriels philanthropes de l'époque, Dale fait construire une école pour les enfants de l'usine, et souhaite offrir à ses ouvriers ainsi qu'à leurs familles des conditions de vie et de travail supérieures à la moyenne de l'époque. À partir de 1800 et jusqu'en 1825, la filature passe sous la direction de Robert Owen, industriel originaire du Pays de Galles et gendre de Dale. Owen ne se contente pas de poursuivre la gestion philanthropique de son beau-père, il se veut à la fois capitaine d'industrie et réformateur social. Nourri de thèses déterministes et d'un idéal de perfectibilité hérité des Lumières, il conçoit New Lanark non comme un village industriel parmi d'autres, mais comme le lieu d'une véritable expérience scientifique. Anciennement directeur d'usine à Manchester, Owen souhaite prouver une intuition nourrie durant ses années passées au contact du monde ouvrier, à savoir que le caractère de l'homme est formé pour lui et non par lui ${ }^{1}$. Il s'agit d'améliorer en profondeur l'environnement direct des ouvriers afin de résoudre de façon définitive les difficultés de leur condition. En vingt-cinq ans, l'expérience de New Lanark passe ainsi par des mesures d'hygiène plus draconiennes, par la réduction des heures de travail

1. Idée qu'Owen exprime pour la première fois par écrit dans A new view of society (1813). 
des ouvriers âgés de 10 à 18 ans et surtout, par un programme d'éducation novateur, allant bien au-delà des attentes de l'époque en matière d'éducation des classes populaires. Destinée aux enfants des deux sexes, et ce dès l'âge de trois ans, l'école de New Lanark propose un programme incluant les « $3 R s$ », mais également des cours d'histoire, de géographie, de sciences naturelles, de chant et de danse, et se caractérise par un refus absolu des châtiments corporels. Attirant l'attention des dirigeants et des réformateurs de l'époque, New Lanark et Owen accèdent à une notoriété internationale, et le village industriel devient une destination touristique en vogue, la visite des écoles constituant le clou du spectacle. Au fil des ans, les idées d'Owen s'affinent et se radicalisent pour aboutir, dans les années 1820, à la formation de l'un des premières doctrines socialistes modernes - l'owénisme. Il ne s'agit plus désormais de réformer la classe ouvrière, mais bien la société toute entière, selon un mode d'organisation sociale communautaire, largement inspiré du système de New Lanark. En 1825, Owen quitte son poste de directeur pour se consacrer entièrement à ses activités politiques, et depuis lors, il est considéré à la fois comme le père du socialisme britannique et comme l'un des pionniers de l'Infant School Movement.

On sait cependant peu que les éléments ayant fait le succès de New Lanark, à savoir sa gestion philanthropique, ses écoles et son organisation communautaire solidaire, étaient déjà largement en place du temps de Dale. C'est là un aspect sur lequel Owen, avec l'orgueil et les talents de publiciste qui sont les siens, n’a guère insisté dans ses écrits. Si Dale n’a pas eu d'ambitions sociales aussi vastes que celles d'Owen, sa politique a en revanche eu une grande influence sur son gendre ${ }^{2}$.

Or cette politique d'entrepreneur philanthrope, et le caractère ainsi conféré au village et à l'entreprise, sont indissociablement liés au contexte écossais qui a vu naître la filature dans les années 1780. Souhaitant faire de New Lanark une communauté à part entière et non un simple appendice à l'usine, David Dale y a directement transposé les structures de la communauté rurale écossaise traditionnelle, et en particulier le système de gouvernement collégial de l'assemblée des elders et la parish school. Plus généralement, nous analyserons dans quelle mesure le climat d' "économie morale » propre à la société écossaise des Lumières finissantes a pu constituer un terrain favorable à l'apparition du village ouvrier « modèle », d'inspiration philanthropique et conçu a priori comme une alternative humaniste aux bouleversements socio-économiques induits par les nouvelles conditions de vie et de travail apparues avec la révolution industrielle. Enfin, il nous faudra examiner l'influence possible ou avérée

2. McLaren (1983), p. 4. 
de ce contexte écossais sur les politiques entrepreneuriales d'Owen, ainsi que sur la formation de sa pensée politique et sociale, d'autant que les spécialistes d'Owen s'accordent pour voir dans New Lanark la matrice de l'owénisme.

Cependant, cette dimension historique et régionale est largement absente de l'historiographie traditionnelle du mouvement owéniste, alors même que New Lanark y occupe une place centrale.

Cette omission s'explique par le fait que la tradition universitaire s'est avant tout intéressée aux aspects politiques et philosophiques de l'owénisme, au détriment de ses origines historiques et sociales. Depuis le Manifeste communiste, Owen est considéré comme l'un des représentants du «socialisme utopique », aux côtés des théories de Fourier et Saint-Simon. Cependant, et c'est là un aspect peu connu de leur argumentaire, Marx et Engels ne rejettent pas entièrement l'owénisme. S'ils récusent son refus de l'action révolutionnaire, assimilé à une incapacité à reconnaitre la lutte des classes comme moteur de l'histoire, ils reconnaissent cependant à Owen un rôle de pionnier, et voient en lui l'un des premiers critiques des conséquences sociales de la révolution industrielle. Cette conception de l'owénisme, qui fait office de canon, a donné naissance à deux courants historiographiques distincts sans être exclusifs l'un de l'autre. Ainsi, ce mouvement a été envisagé soit dans la perspective d’une histoire générale du socialisme, soit dans celle de l'utopie et de la pensée utopique. Mais dans les deux cas, Owen est toujours envisagé comme le «père du socialisme britannique ». Popularisée par Frank Podmore, membre fondateur de la Société Fabienne et premier biographe d'Owen, cette lecture a ensuite été reprise par les historiens proches des mouvements socialistes non révolutionnaires (notamment les fabiens et les coopératistes) et, dans une moindre mesure, par un certains nombre d'universitaires associés au marxisme et à la New Left ${ }^{3}$.

Selon J. F. C. Harrison, l'un des plus brillants commentateurs d'Owen et de l'owénisme, cette interprétation fait aujourd'hui encore figure d'orthodoxie ${ }^{4}$. D'un point de vue politique tout d'abord, la faillite des communautés owénistes proprement dites, telles New Harmony ou Orbiston, a été laissée de côté pour insister sur l'expérience réussie de New Lanark.

3. Frank Podmore, Robert Owen : A Biography (2 vols.), London, Allen \& Unwin, 1906. Parmi les historiens voyant en Owen la figure tutélaire du socialisme britannique, voir Max Beer, The History of British Socialism, London, G. Bell \& Sons, 1919; William Postgate, The Builders' History, Londres, The Labour Publishing Company, 1923, et surtout G. D. H. Cole, The Life of Robert Owen, Londres, Macmillan, 1930. S'il critique vivement certains aspects utopiques de la pensée d'Owen, E. P. Thompson lui reconnaît cependant, à l'instar de Marx et Engels, un rôle précurseur dans le développement du socialisme britannique.

4. Harrison (1969). 
Seul village industriel à avoir inspiré un modèle social alternatif, New Lanark est considéré à la fois comme un haut lieu du mouvement coopératif, dont Owen serait également le père fondateur, et comme un avant-goût des politiques du welfare state mises en place par les gouvernements travaillistes au vingtième siècle. D'un point de vue social, on retient surtout la contribution d'Owen au progrès de l'éducation populaire, cet aspect constituant le volet le moins « utopique » de sa pensée. Tenu pour être le fondateur de l'Infant School Movement et le pionnier de l'école moderne, Owen serait, en quelque sorte, le Jules Ferry britannique. Sous cet angle presque exclusivement politique, New Lanark est envisagé comme une utopie socialiste et/ou comme le lieu fondateur du socialisme britannique. Ce faisant, on perd de vue que New Lanark était avant tout une communauté industrielle, quand bien même de nature alternative. Comme le fait justement remarquer David J. McLaren, peu d'auteurs ont rattaché l'œuvre d'Owen au contexte particulier de l'industrie textile naissante, pour voir en lui un innovateur purement idiosyncratique ${ }^{5}$. Ce manque de contextualisation nuit d'autant plus à la compréhension de New Lanark et de l'owénisme que la source de référence demeure l'autobiographie d'Owen ${ }^{6}$, témoignage problématique s'il en est. Un nombre assez conséquent d'auteurs, à commencer par Frank Podmore, estiment en effet qu'Owen a forcé le trait en exposant de manière exagérément négative le New Lanark de David Dale, afin de mieux mettre en valeur les aspects de sa propre gestion philanthropique. Cette critique, bien que relativement présente, n'est cependant pas parvenue à concurrencer véritablement la tentation de l'idéalisme dont fait preuve l'historiographie owéniste classique ${ }^{7}$. À la suite d'Owen lui-même, de nombreux commentateurs continuent notamment de présenter Owen comme le fondateur des écoles de New Lanark, et en particulier de son école maternelle, alors que ces institutions ont été mises en place du temps de Dale, et ce dès la création du village. À ce jour, dans le lignage des travaux de J. F. C. Harrison, seuls quelques auteurs, écossais pour la plupart, se sont attachés à replacer New Lanark dans son contexte historique et régional, notamment en ce qui concerne la filiation entre les écoles du village et les parish schools écossaises, et l'influence de la foi calviniste de Dale sur sa politique entrepreneuriale éclairée. Les structures sociales du villages, fortement inspirées de celles de la paroisse presbytérienne, restent quant à elles à explorer.

5. McLaren (2003), p. 3.

6. Owen (1857).

7. À tel point qu'Owen est parfois désigné comme le fondateur de New Lanark, comme on peut le lire sur le site de la British Library. 


\section{Origines du village ouvrier}

Loin d'être une utopie socialiste, New Lanark doit être analysé, tout comme les autres communautés industrielles du même type, en tant que village ouvrier. Ces agglomérations de taille modeste, créées totalement ou presque par l'industriel autour de la mine ou de l'usine, elles diffèrent donc des villes industrielles, telles Glasgow ou Manchester qui, à un moment donné de leur histoire, ont opéré une spécialisation dans le secteur secondaire ${ }^{8}$. Les premiers villages ouvriers textiles apparaissent dans la vallée de la Derwent (Derbyshire) à la fin du dix-huitième siècle. En 1771, Richard Arkwright, industriel et inventeur du métier à filer hydraulique, fonde la filature de Cromford afin de mettre son water-frame à l'épreuve. L'usine, implantée en bord de rivière, se voit progressivement dotée d'une cité ouvrière, afin d'attirer et de fixer la main d'œuvre sur place. Les usines de type Arkwright sont d'abord imitées localement. Trois autres villages ouvriers sont construits dans la même vallée par des associés d'Arkwright tels que Samuel Greg, qui fonde la filature de Styal en 1784. Par la suite, le système s'implante dans le reste de la Grande-Bretagne. En Écosse, Arkwright s'associe à Dale pour fonder New Lanark en 1785, et fait de Cromford un centre de formation destiné à initier ouvriers et mécaniciens au fonctionnement du water-frame. Outre New Lanark, au moins huit autres communautés industrielles de type Arkwright sont créées en Ecosse dans la vallée de la Clyde, le Perthshire, l’Ayrshire et les Highlands.

Cet essor n'est pas le fruit du hasard. Arkwright a cherché à développer ses activités au-delà du Tweed en raison d'une guerre des patentes que lui livrent ses concurrents anglais. Les villages industriels précités naissent tous d'un partenariat entre Arkwright et les élites industrielles locales, à qui il fournit non seulement ses water-frames, mais aussi un programme d'apprentissage. Comme leurs homologues de la vallée de la Derwent, menuisiers et mécaniciens écossais effectuent un séjour à Cromford avant l'ouverture de leur usine, afin de se former à la construction, au maniement et à l'entretien des machines ${ }^{9}$. Côté écossais, Arkwright peut compter sur une abondance de cours d'eau puissants, sur une importante main d'œuvre - pour la plupart des paysans devenus sans terre au lendemain des Highland Clearances - ainsi que sur une volonté de la part des industriels écossais de voir Glasgow supplanter Manchester dans le contrôle du secteur textile. D'un point de vue institutionnel, les villages-usines ne sont pas sans rappeler la vogue des villages nouveaux (planned villages) que

8. Simon Edelblutte (2008).

9. <http://www.derwentvalleymills.org/> 
connait l'Écosse à la même époque. Entre 1730 et 1830, environ 150 villages sont créés ex nihilo ou largement remaniés par la noblesse locale, afin de bonifier et d'enclore les terres dans le cadre de l'essor de l'agriculture commerciale. Au fil du temps, et à mesure que s'affirme le développement du secteur textile en Grande-Bretagne, des villages nouveaux dédiés à la manufacture de la laine et du lin ou à la filature du coton voient le jour, comme à Stanley (Perthshire), appelé de ses vœux par la noblesse locale afin de parachever le développement économique local. Il en va de même pour New Lanark, dont l'établissement et le droit d'accès aux ressources en eau se fait avec l'accord des notables locaux, qui y perçoivent à juste titre une occasion de diversification des activités dans le Lanarkshire ${ }^{10}$.

En Écosse, les villages ouvriers textiles, et New Lanark avec eux, se trouvent à la conjonction du succès du système d'Arkwright et d'une tradition écossaise de planification communautaire en milieu rural. En outre, comme au sein des usines éclairées, ces planned villages mêlent d'emblée des préoccupations économiques, esthétiques et sociales. Un soin particulier est en effet accordé à l'architecture du lieu, tandis que le village est doté d'une mission à la fois humanitaire et civilisatrice. Beaucoup d'entre eux, tels Stanley ou encore les pêcheries d'Ullapool, ont en effet été expressément bâtis afin d'accueillir et d'employer des communautés paysannes déplacées par les enclosures et les clearances, souvent par ceux-là mêmes à avoir ordonné leur éviction ${ }^{11}$.

Les villages ouvriers ont en commun un ensemble d'éléments architecturaux. Au lieu de travail s'ajoute une cité ouvrière attenante, sous forme d'habitat individuel ou collectif, ainsi que des bâtiments à vocation sociale : école, magasin, voire, dans les cas les plus poussés, dispensaire, friendly society, bains, lavoirs, parcs, etc. Fruit d'une gestion paternaliste, chaque village-usine est par conséquent le reflet de la personnalité de son fondateur, d'où de nombreuses variations d'un cas à l'autre. Néanmoins, dans la majorité des cas, ces villages deviennent des modèles de gestion industrielle éclairée, avec une volonté plus ou moins forte et plus ou moins consciente de se poser en alternative à la grande usine urbaine et aux conséquences socio-économiques de la révolution industrielle sur les classes ouvrières.

10. Donnachie et Hewitt (1993), chapitre 2.

11. T. C. Smout (1970), p. 73-100. 


\section{Organisation interne du village de New Lanark}

La vie de l'usine étant intrinsèquement liée à celle du village et vice versa, la création d'un réseau d'institutions de proximité n'est pas une simple coquetterie de la part des industriels philanthropes. Il s'agit avant tout d'établir un sentiment de communauté nécessaire pour assurer la viabilité du village ouvrier. Dans cette perspective, il n'est pas étonnant que, sous l'impulsion de son fondateur, l'organisation interne de New Lanark se soit largement inspirée des structures traditionnelles du village écossais des Lowlands, et notamment de ses institutions emblématiques : la Kirk Session et l'école paroissiale. Même si l'autorité du chef d'entreprise est indéniable, la présence d'un réseau d'institutions et de modes de sociabilité à l'origine extérieurs au monde de l'entreprise montre que le village ouvrier ne fonctionne pas en complète autarcie.

Tout d'abord, les structures communautaires et administratives de New Lanark sont calquées sur le fonctionnement traditionnel des paroisses écossaises. Depuis la réforme knoxienne des années 1560, chaque paroisse est dirigée par la Kirk Session, assemblée composée du pasteur et des elders, des notables laïcs nommés par les paroissiens et chargés de les représenter. La Session est en charge de la justice locale, d'une mission de veille morale et spirituelle ainsi que de l'application des Poor Lawes. Dès sa création, New Lanark est rattaché à la paroisse la plus proche, celle du bourg de Lanark, situé à environ deux miles en amont du village ouvrier. Par conséquent, la Kirk Session locale examine les cas de mariages irréguliers, d'adultère et de naissances illégitimes à Old Lanark comme à New Lanark, et les deux localités figurent sur les mêmes registres paroissiaux. Néanmoins, dans la mesure où Dale contribue financièrement au bien-être matériel de « ses » pauvres en leur fournissant un emploi, un toit, une école et une chapelle, il est dispensé du paiement des poor rates ${ }^{12}$. Ces liens se maintiennent du temps d'Owen : en 1817, quatre employés de New Lanark sont ordonnés elders, ce qui parachève l'inclusion du village ouvrier dans sa paroisse ${ }^{13}$.

Outre cette intégration à sa paroisse de rattachement, New Lanark a mis en place son propre système de gouvernement collégial. Le village était découpé en « divisions de voisinage » (neighbourhood divisions), qui élisaient douze représentants, ou jurors, chargés de faire le lien avec la direction, de recueillir les doléances de la population et de statuer sur les affaires d'ordre au sein de la communauté. S'il est difficile de savoir qui de Dale ou d'Owen a mis ce système en place, la ressemblance avec l'organisation et les fonctions de la Kirk Session est néanmoins frappante. Dans

12. Lanark Heritors Minute Books, 1816-1828.

13. Lanark Old Parish Records 1786-1807, New Lanark Conservation Trust. 
les deux cas, il s'agissait avant tout de maintenir une cohésion morale et sociale à l'échelle de la communauté, et d'entériner les structures paroissiales en place. On peut néanmoins penser que Dale, qui occupait également des fonctions de pasteur, a pu avoir son rôle à jouer dans la création de ces neighbourhood divisions. Calviniste fervent, il était en effet l'un des membres fondateurs d'une secte presbytérienne dissidente, les Old Scotch Independents, au sein de laquelle il avait pour habitude de prêcher depuis les années 1770, sans pour autant avoir été ordonné. Ces prêches avaient lieu aussi bien à Glasgow, dans la chapelle des Independents, qu'à New Lanark, à destination de la population du village ${ }^{14}$. En dotant son entreprise d'une cité ouvrière et d'une chapelle, où il officie de surcroît, et en calquant son modèle de communauté sur la tradition du village écossais, Dale venait donc se substituer à l'autorité économique et politique de la noblesse terrienne et à celle, morale, du clergé.

Au sein de cette communauté, le rôle des écoles était particulièrement saillant, dans la mesure où il s'agit d'enraciner sur place une main d'œuvre majoritairement non-autochtone. À côté d'un petit nombre d'employés originaires des environs immédiats, l'entreprise employait de nombreux apprentis venus des workhouses de Glasgow et d'Édimbourg, ainsi que des Highlanders venus, comme ailleurs dans les Lowlands, chercher un emploi au lendemain des clearances. Les écoles de New Lanark constituent sans doute l'aspect le plus connu et le plus étudié de l'histoire du village. On connait surtout l'Institut pour la Formation du Caractère fondé par Owen en 1816, mais celui-ci s'appuie largement sur les acquis de l'époque de Dale. Les deux systèmes, en effet, reposent sur une même tripartition entre école maternelle pour les plus jeunes, école primaire pour les enfants travaillant à l'usine, et enfin, une Sunday school dédiée à l'enseignement du catéchisme. Si elle est moins connue, l'influence de Dale quant à la création de ces écoles est cependant avérée. De même que pour l'organisation du village Dale, et Owen après lui, ont largement appuyé leur politique éducative sur le contexte écossais de leur temps ${ }^{15}$. Les écoles de New Lanark sont en effet à envisager comme une transposition en milieu industriel du système des parish schools, caractéristique des campagnes des Lowlands ${ }^{16}$.

Nées elles aussi de la réforme knoxienne, les parish schools constituent l'un des piliers de la tradition presbytérienne écossaise. Financées par la

14. Thomas Garnett, Observations on a Tour through the Highlands, 1800, p. 235; cité dans McLaren (1983), p. 61-62.

15. McLaren (2003), p. 3.

16. Le système des écoles paroissiales ne s'appliquait pas en ville et dans les Royal Burghs, où l'éducation était prise en charge par les autorités municipales. 
noblesse locale, elles étaient supervisées par la Kirk Session, qui nommait et payait les instituteurs et approuvait le contenu des cours. Celui-ci était plus large que dans le reste de la Grande-Bretagne, incluant non seulement les « 3 Rs », mais aussi des leçons de chant, de catéchisme et de latin. En outre, ces écoles étaient ouvertes aux garçons comme aux filles, ce qui conférait à l'Écosse des Lumières un taux de scolarité supérieur au reste de l'Europe ${ }^{17}$. Enfin, elles étaient payantes, sauf pour les enfants relevant des Poor Lawes. Entre la fin du dix-huitième et le début du dixneuvième siècle, on comptait environ 900 parish schools en Écosse, où étaient scolarisés $20 \%$ des enfants du pays ${ }^{18}$.

La transposition du système à New Lanark a sans doute été facilitée par le fait que Dale avait lui-même effectué sa scolarité dans la parish school de sa ville natale, Stewarton (Ayrshire) ${ }^{19}$. Sa connaissance de ce système explique en partie la ressemblance frappante entre les écoles paroissiales et celles de New Lanark. La scolarité y était également payante, hormis pour les enfants les plus pauvres du village — soit des orphelins employés comme apprentis par Dale, soit les enfants de moins de six ans, encore trop jeunes pour travailler et gagner leur vie. L'école était mixte, et financée par David Dale, qui venait se substituer ainsi à l'action conjointe de la noblesse locale et de la Kirk Session. Enfin le programme était calqué sur celui des parish schools et incluait les « 3 Rs », ainsi que des leçons de catéchisme et de musique sacrée, comme en témoigne le médecin et scientifique Thomas Garnett lors d'une visite à New Lanark :

On going in to the day school we heard some little boys read in a superior manner. In the evening these three masters are assisted by seven others, one of whom teaches writing. [...] At dinner the masters preside over the boys at table, performing the office of chaplains and conduct them on Sundays to divine worship [...]. In the evening of Sunday, all the masters attend to teach and give religious instruction ${ }^{20}$.

Ce texte nous montre également que, tout comme leurs homologues des parish schools, les instituteurs de New Lanark cumulaient des fonctions d'enseignant, d'aumônier et de maître de chœur. Enfin, la Kirk locale avait également un droit de regard sur les écoles de New Lanark, droit dont elle ne s'acquitte formellement qu'à partir de 1823, au moment où

17. McLaren (2003), p. 4.

18. D. Myers, «Scottish Schoolmasters in the Nineteenth Century: Professionalism and Politics ", dans W. Humes et J. Paterson (1983), p. 76-80; T. C. Smout (1969), p. 423; McLaren (2003), p. 8-9.

19. Robert Chambers (1856), p. 162.

20. Thomas Garnett (1800), p. 235, cité dans McLaren (1983), p. 61-62. 
Owen est soupçonné par ses associés de vouloir bannir l'enseignement du catéchisme ${ }^{21}$.

Si les écoles de New Lanark reprenaient les modes d'organisation et d'enseignement de la parish school, elles en avaient également conservé l'esprit. L'intérêt de Dale pour l'éducation des enfants de New Lanark est donc à analyser en rapport avec l'importance accordée à l'instruction élémentaire tant dans la tradition presbytérienne que dans le discours patriotique écossais qui se développe au lendemain de l'Acte d'Union.

Pour les presbytériens, suivant les préceptes de John Knox exprimés dans le First Book of Discipline (1560), la création d'écoles dans chaque paroisse constituait une nécessité absolue, et ce dans trois buts précis : l'évangélisation du peuple, en particulier dans les paroisses rurales les plus reculées, l'inculcation d'un code moral strict et vertueux pour la jeunesse du pays via l'étude des textes sacrés et enfin, la réalisation d'un acte de charité envers les plus pauvres ${ }^{22}$. Le salut du peuple écossais reposait donc sur un idéal d'éducation universelle, incarné par la parish school. Pour Tom Devine, l'éducation élémentaire telle qu'elle était dispensée dans ces écoles constituait « the means by which the essential precepts of religious beliefs were to be instilled in the young ${ }^{23}$ ». Calviniste fervent, devenu presbytérien évangélique dès l'adolescence lors des réveils de Cambuslang $^{24}$, Dale a mis en place à New Lanark une politique éducative dans le droit fil des préceptes knoxiens — par la nature des programmes scolaires et l'attention particulière portée à l'enseignement du catéchisme, par l'injonction morale à favoriser l'éducation des plus pauvres et enfin, par l'acte même de fondation des écoles.

Au lendemain de l'Acte d'Union, l'idéal presbytérien d'éducation universelle se charge progressivement de connotations patriotiques également présentes à New Lanark. En tant qu'institution phare de l'identité écossaise avec la Kirk et le système judiciaire, l'école acquiert une signification symbolique fondamentale dans les milieux nationalistes, dont Dale fait partie. Dans son autobiographie, Robert Dale Owen, fils aîné de Robert Owen et d'Anne Caroline Dale, raconte ainsi l'attachement de son grand-père au Scots, qu'il emploie systématiquement pour s'adresser à ses ouvriers. Fervent patriote, c'est également un admirateur de la culture gaélique, qu'il connaît via son épouse, issue du puissant clan Campbell. Enfin, sa méfiance originelle à l'égard d'Owen, et ses réticences à lui accorder la main de sa fille, sont dues non seulement au déisme de son

21. Anonyme, One Formerly A Teacher at New Lanark (1839), p. 15.

22. John Knox, The First Book of Discipline (1560), dans William M'Gavin (1831), p. 498.

23. T. M. Devine (1999), p. 9; cité également dans McLaren (2003), p. 6.

24. Chambers (1856), p. 163. 
futur gendre, mais aussi à son statut d' "Anglais ${ }^{25} »$. Au cœur du discours patriote, on retrouve notamment le mythe du «lad o'pairts », ce garçon d'origine modeste qui, grâce à ses talents et à l'excellence de la parish school, parvient à s'élever dans la société, notamment grâce à l'enseignement du latin, qui ouvrait à l'époque les portes de l'université.

Les écoles de New Lanark, destinées à des enfants d'ouvriers et à de jeunes travailleurs, ne visaient pas aussi haut en terme d'idéal de mobilité sociale, mais elles allaient cependant au-delà des normes de l'époque, qui prescrivaient une instruction minimale pour les classes ouvrières, à la mesure de leur rang supposé. En tant que self-made man, on peut penser que Dale a été sensible à cet idéal du lad o'pairts et qu'il a tenté de l'adapter au monde de la filature de coton et du village ouvrier. Fils d'un épicier de village, il est dès l'enfance employé comme gardien de troupeaux, avant d'être placé en apprentissage dans un atelier textile. Peu à peu, il gravit les échelons, devenant intermédiaire auprès des négociants en draps de Glasgow puis importateur de toile de lin, avant de s'établir à son compte comme industriel ${ }^{26}$. Et de fait, au-delà de la nécessité d'éducation religieuse et morale de la jeunesse dictée par la doctrine presbytérienne, l'éducation à New Lanark ne constitue ni un vœu pieux, ni une fin en soi. Il s'agit de préparer l'avenir professionnel des enfants, et en particulier des apprentis, une fois leur contrat achevé. Dans une lettre datée de 1790, Dale exprime son désir de donner aux élèves « les moyens de gagner leur pain » une fois ces derniers sortis de l'usine ${ }^{27}$. Il semble que cette volonté ait porté ses fruits. Même Owen, pourtant peu enclin à louer son beaupère, concède publiquement que, si les enfants travaillaient dur, comme dans l'ensemble des usines de l'époque, ils étaient cependant « extremely well fed, well clothed, well lodged, and very great care taken of them when out of the mills ${ }^{28}{ } »$. De fait, Dale met la parish school à la portée des enfants les plus pauvres, et leur fournit ainsi un niveau d'instruction bien supérieur à celui dispensé dans les charity schools qu'hébergeaient les workhouses et hôpitaux du pays ${ }^{29}$. Ce faisant, il va plus loin que ce qui est attendu de lui par les agents chargés du placement des parish apprentices. Par exemple, les minutes de la Kirk Session de la paroisse de Corstorphine, près d'Edimbourg, qui envoyait de nombreux enfants à New Lanark,

25. Robert Dale Owen (1874), p. 36 et 54-55.

26. McLaren (1983), chap. 1.

27. "I think I can promise to put them all in a way of gaining their bread », Lettre de David Dale à R. Richardson, 7 septembre 1790; cité dans McLaren (1983), p. 68.

28. House of Commons Parliamentary Papers, 1816 (397), Report of the minutes of evidence, taken before the Select Committee on the State of the Children Employed in the Manufactories of the United Kingdom, témoignage de Robert Owen, p. 20.

29. McLaren (2003), p. 12. 
n'exigeaient des industriels qu'une instruction minimale, limitée à des cours de lecture et de catéchisme ${ }^{30}$. En outre, Dale est allé plus loin encore que les propriétaires d'autres villages ouvriers, où l'éducation des enfants se faisait le plus souvent dans une Sunday school. Enfin, il a innové avec la création d'une garderie pour les moins de six ans, afin de permettre à ses ouvrières d'allier travail et vie de famille ${ }^{31}$. Si Owen a entrepris de systématiser la politique d'éducation à New Lanark, l'intégrant à sa propre philosophie déterministe, c'est donc bien à Dale que l'on doit ce qui est sans doute la première école maternelle au monde, contrairement à ce qu'Owen et ses disciples n'ont cessé d'affirmer. Certes, Owen a mis en place dans ses écoles un programme scolaire beaucoup plus large, davantage inspiré des grammar schools destinées aux classes aisées que de la parish school écossaise. De plus, ces écoles étaient l'expression de ses propres théories, elles-mêmes porteuses d'influences qui dépassent le cadre écossais, telles que la pensée de Locke, Rousseau ou encore Pestalozzi. Cependant, Owen conserve la division tripartite entre école maternelle, primaire et Sunday school déjà présente du temps de Dale. En dépit de ses réserves personnelles, il ne supprime pas les cours de catéchisme, conformément aux attentes des ouvriers de New Lanark, tous profondément religieux ${ }^{32}$. Et enfin, comme à l'époque de Dale, les écoles d'Owen conservent leur rôle de ciment identitaire et social, et deviennent un lieu de célébration de la culture écossaise, où l'on enseigne l'histoire, les chants et les danses traditionnels du pays, et où les garçons sont encouragés à porter le kilt ${ }^{33}$.

\section{Lumières écossaises et économie morale}

New Lanark et les villages ouvriers sont plus qu'une simple transposition des structures villageoises en milieu industriel, plus qu'une simple usine à la campagne.

Pourquoi, en effet, investir dans un village ouvrier, très coûteux à mettre en place en comparaison avec une usine urbaine? Au-delà de la promesse d'importants retours sur investissement, la création d'un tel type d'entreprise entre en résonance avec les convictions personnelles de leurs fondateurs. Nombre d'entre eux, Dale y compris, sont des self-made men, souvent d'anciens employés du textile, de fait sensibles aux réalités

30. Corstorphine Kirk Session Minutes, April 5th 1796, National Archives of Scotland, NAS $\mathrm{CH} 2 / 124 / 4$.

31. McLaren (2003); Statistical Accounts of Scotland, Lanark, 1795, <http://edina.ac.uk/statacc-scot/ $>$.

32. McLaren (2003), p. 20-21.

33. Owen (1957), chap. 5. 
du monde des artisans et des ouvriers. En outre, l'écrasante majorité des fondateurs de villages ouvriers appartiennent à des sectes protestantes dissidentes, dont l'intérêt pour la philanthropie a été largement documenté ${ }^{34}$. Dale ne fait pas exception à la règle, et développe une rhétorique de l'entreprise vertueuse, où santé financière et bien-être physique, moral et spirituel de la main d'œuvre sont indissociables. Dès avant Owen, Arkwright et ses disciples considèrent donc, à des degrés divers, le village ouvrier comme une alternative à l'usine urbaine. New Lanark et les villages ouvriers sont donc à replacer dans un double contexte, celui du renouveau évangélique du milieu du dix-huitième siècle et celui des Lumières finissantes.

Présent lors des réveils de Cambuslang en 1742, qui marquent sa conversion à la foi évangélique, Dale est un ardent partisan du Reform Movement. Bailleur de fonds de l'hôpital de Glasgow, il est également connu pour distribuer de la nourriture aux habitants de la ville en période de disette. Son souhait de fournir des conditions de vie et de travail décentes à sa main d'œuvre est en lien avec son engagement religieux, mais également à l'esprit des Lumières écossaises, qui mêle idéal de perfectibilité, importance accordée au sujet mais aussi crainte du chaos social. Le village-usine se doit donc d'être un îlot d'ordre et de raison au sein d'un monde de désordre. Cet esprit des Lumières est également à rapprocher d'une volonté, présente chez les industriels philanthropes, d'humaniser l'économique. Dans La grande transformation, Karl Polanyi a montré que l'idée selon laquelle le marché existe et se régule de manière autonome n'apparaît pas avant les années $1830^{35}$. Avant cela, les commentateurs défendent une conception de ce que Polanyi nomme l'encastrement (embeddedness) du marché dans la société civile. D'après cette même conception, les lois du marché sont soumises à celle de la communauté. Si l'enrichissement personnel est souhaitable et valorisé dans un pays de tradition calviniste, il reste néanmoins inféodé à des finalités plus hautes, d'inspiration religieuse, qui sont la préservation de la société et de la communauté, le bien-être individuel et collectif étant indissociables. David Dale, dans l'une des rares sources écrites émanant de lui, ne dit pas autre chose :

[...] we should aim at glorifying God and doing good to ourselves and others. Diligence in business matters is a duty; but in this and in all things, we should be 'fervent in spirit', serving the $\operatorname{Lord}^{36}$.

34. Sur le rapport entre évangélisme, philanthropie et patronat, voir notamment Hilton (2001), chap. 4-6.

35. Polanyi (1944).

36. Substance of a Discourse by David Dale, janvier 1792. 
On retrouvera chez Owen cette même volonté, sous une forme sécularisée cette fois-ci, d'humaniser l'économique, cette même tentative, caractéristique fondamentale de la pensée des Lumières, de remettre en ordre un monde perçu comme chaotique. De ce fait, la cause de la misère sociale n'est pas l'industrialisation elle-même, mais la manière égoiste et irrationnelle dont elle a jusqu'alors été menée :

[...] we cannot return to our former state; for without the cotton trade, our increased population cannot be supported, the interest of the national debt paid, nor the expenses of our fleets and armies defrayed. Our existence as an independent power now, I regret to say, depends on the continuance of this trade, because no other can be substituted in its place. True indeed it is, that the main pillar and prop of the political greatness and prosperity of our country is a manufacture which, as it is now carried on, is destructive to the health, morals, and social comforts of the mass of the people engaged in it ${ }^{37}$.

Avec l'Acte d'Union de 1707, cette conception de l'économie et du marché prend des colorations patriotiques par l'importance qu'elle confère au lien social. Le sentiment national écossais se cristallise sur les institutions emblématiques de la nation (l'Église presbytérienne, le système judiciaire et scolaire) mais également sur les institutions de proximité que sont la famille, la paroisse et la communauté villageoise. Le discours sousjacent est le suivant : si la société écossaise est plus pauvre que la société anglaise, elle est cependant plus vertueuse, n’ayant pas sacrifié le lien social à la modernité économique; le village-usine en est une des manifestations, vanté par les auteurs des Statistical Accounts of Scotland comme la preuve tangible de l'avancement économique et moral du pays, puisqu'il allie bonne santé économique et relations de classe apaisées. L'extrait suivant, consacré au village industriel de Huntly (Aberdeenshire) est représentatif de cette tendance :

The village $[\ldots]$ has surprisingly increased within these fifty years, in population and industry, in so much that where all around for some distance was formerly barren heath, swamps or marsh there is now scarcely one uncultivated spot to be seen [...]. This spirit of improvement and manufacture was first introduced by a few who dealt in the yarn trade. From their laudable example and from observing the profits arising from industry, others were encouraged; and now it has become one of the first villages in the North ${ }^{38}$.

37. Robert Owen, Observations on the cotton trade, with a view to the intended application to Parliament for a repeal of the duties on the importation of cotton wool (January 1815), reproduit in Owen, 1858, Appendix F, p. 16.

38. Statistical Accounts of Scotland, VI, 1793, p. 129, dans Smout (1970), p. 77. 
Comme les autres industriels philanthropes de son pays, Dale voit dans le village-usine un véritable remède aux maux de son pays. De fait, comme le montrent les nombreuses offres d'emplois qu'il fait publier dans la presse, le recrutement se tourne de façon volontaire vers les couches les plus défavorisées de la société écossaise de l'époque :

Families from any quarter possessed with a good moral character and having 3 children fit for work, above 9 years of age, are received - supplied with a house at a moderate rent, and the women and children provided with work ${ }^{39}$.

Outre les femmes (veuves ou non) avec enfants, Dale emploie un grand nombre d'orphelins, auxquels il souhaite donner les qualifications nécessaires pour échapper à la double menace des poor reliefs et de la workhouse ${ }^{40}$. Mais surtout, via son alliance avec le clan Campbell, il fait jouer ses réseaux familiaux pour embaucher de nombreux Highlanders victimes des Clearances. Dale perçoit en effet le travail en usine comme le seul antidote à l'émigration de masse des habitants des hautes terres vers les États-Unis, qu'il considère comme une véritable catastrophe économique et sociale pour l'Écosse :

Could the people find employment in the Highlands, it would be much better for them to remain here; but as it is not the case the best thing that can be done for them and for this country is to invite all that cannot find employment to come here [New Lanark] and they will be provided for ${ }^{41}$.

Cette politique mêlant patriotisme, intérêt économique lié à l'emploi d'une main d'œuvre bon marché et altruisme était relativement répandue dans les milieux éclairés de l'époque, où se côtoient essayistes, réformateurs, fondateurs de planned villages et industriels philanthropes. Les acteurs des Lumières écossaises s'alarment généralement du nombre croissant d'émigrants en partance pour les États-Unis ${ }^{42}$. La Society for Preventing Emigration to Foreign Parts est fondée en 1791, et Dale en devient l'un des membres, aux côtés d'autres fondateurs de villages ouvriers. Dans la continuité de l'idéal de perfectibilité économique incarné par les planned villages agricoles, l'avenir de l'Écosse dépend de l'implantation de filatures en milieu rural en général, et dans les Highlands en particulier. Par exemple, le duc d'Atholl fonde le village ouvrier de Stanley (Perthshire) dans le but précis de trouver un logement et un emploi nouveaux

39. Lettre de Dale à la presse, dans Harrison (1969), p. 52

40. Donnachie et Hewitt (1999), p. 39-40.

41. Lettre de David Dale au Scots Magazine, octobre 1791. Cité dans Donnachie et Hewitt (1999), p. 39 .

42. Donnachie et Nicolson, p. 24. 
à des communautés de crofters déplacées dans le cadre du mouvement des enclosures. Dale participe également à la fondation de deux autres villages ouvriers situés dans les Highlands, à Ballindaloch et Spinningdale (Sutherland).

De façon significative, toutes les filatures rurales écossaises seront soit établies dans les Highlands, soit dépendantes d'une main d'œuvre largement originaire de cette région. La fondation de New Lanark et sa composition socioprofessionnelle s'inscrivent donc pleinement dans le contexte écossais de l'époque, au carrefour de la révolution industrielle et de la préservation des intérêts économiques et sociaux nationaux, exigence vitale dans le cadre de l'Union. Cette politique de recrutement préférentielle se poursuivra avec Owen : une annonce publiée dans l'Aberdeen Fournal en 1807 montre qu'à cette époque comme dans les années 1780, on recherchait le même type d'employés potentiels : «Cleanly, industrious and well-behaved families, in which there are three or more children, upwards to ten years of age, natives of the Highlands or Lowlands of Scotland only $[\ldots]^{43} \cdot »$

\section{Conclusion}

C'est avec David Dale plus qu'avec Robert Owen qu'apparaît le premier effort à grande échelle pour humaniser l'usine en industrialisant le village, pour réconcilier tradition morale et modernité économique. New Lanark a ainsi été le lieu d'une alliance entre industrie, communauté et charité plus poussée encore que dans les autres villages ouvriers de l'époque, en raison d'une organisation villageoise communautaire et de l'importance capitale accordée à l'éducation. Certes, Owen est allé plus loin que son prédécesseur, puisque New Lanark lui servira de base pour développer une philosophie politique visant à la réforme de l'ensemble de la classe ouvrière, puis, peu à peu, de la société toute entière. Si Dale n'a pas eu de vision sociale aussi large, l'influence de son appartenance écossaise, qu'il n'a cessée de revendiquer, a joué un rôle essentiel dans la formation de la doctrine de son gendre, qui en proposera une version systématisée et sécularisée.

Au-delà de leurs divergences politiques et religieuses, Dale et Owen avaient plus d'objectifs en commun qu'on ne pourrait le croire. La philanthropie d'inspiration évangélique de l'un et la pensée déterministe et utilitariste de l'autre témoignent toutes deux d'une croyance en l'indissociabilité du bien-être collectif et individuel, et d'une volonté héritée des

43. Aberdeen Journal, 29 avril 1807 (je souligne). 
Lumières écossaises d'humaniser l'économique. Entreprise emblématique de la révolution industrielle naissante comme du renouveau philanthropique et évangélique, New Lanark apparaît comme un véritable creuset, reflet des institutions locales propres à l'Écosse des Lowlands comme d'une identité écossaise originale dans le cadre de l'Union en formation. Pour l'histoire générale de l'industrialisation de l'Écosse comme pour celle du mouvement et de la pensée owénistes, il importe donc de redonner sa place à la dimension spécifiquement écossaise de New Lanark.

\section{Bibliographie}

\section{Sources primaires}

Aberdeen Fournal, 1807.

Corstorphine Kirk Session Minutes, National Archives of Scotland.

Glasgow Mercury, 1791.

Gourock Ropework MSS, Register of Births, Marriages and Deaths, UGD 42/7/5.

House of Commons Parliamentary Papers, 1816 (397), Report of the minutes of evidence, taken before the Select Committee on the State of the Children Employed in the Manufactories of the United Kingdom.

National Archives of Scotland, Lanark Heritors Minute Books 18161828.

National Archives of Scotland, New Lanark Presbytery Records, CH2/ 234.

Scots Magazine, 1791.

\section{Sources secondaires}

Anderson R. D., Education and the Scottish People 1750-1918, Oxford, Oxford University Press, 1995.

Anonyme, One Formerly A Teacher at New Lanark, 1839.

Chambers Robert (éd.), The Biographical Dictionary of Eminent Scotsmen, vol. V., Édimbourg et Londres, 1856.

DaLe David, Substance of a Discourse by David Dale, janvier 1792.

Devine Tom M., The Scottish Nation 1700-2000, Londres, Penguin, 1999.

Donnachie Ian et Hewit George, Historic New Lanark: the Dale and Owen community since 1785, Édimbourg, Edinburgh University Press, 1999 [1993].

Donnachie Ian et Nicolson Margaret, « The New Lanark Highlanders: migration, community and language 1785-c. $1850 »$, Family and Com- 
munal History, vol. VI, n 1,2003 , p. 19-32.

Edelblutte Simon, « Paysages et territoires du patrimoine industriel au Royaume-Uni », Revue géographique de l'Est, vol. 48, n 1-2, 2008, URL : <http://rge.revues.org/1165> [consulté le 14 mai 2011].

Harrison John Fletcher Clewes, Robert Owen and the Owenites in Britain and America: The Quest for the New Moral World, Londres, Routledge \& Kegan Paul, 1969.

HiLton Boyd, The age of atonement: the influence of evangelicalism on social and economic thought 1785-1865, Oxford, Oxford University Press, 2001 [1986].

Humes W. et Paterson J. (éds), Scottish Culture and Scottish Education, Édimbourg, John Donald, 1983, p. 10.

Knox John, The First Book of Discipline (1560), dans William M'Gavin (éd.), The History of the Reformation of Religion in Scotland, Édimbourg, 1831.

Marx Karl et EngeLs Friedrich, Le manifeste communiste, dans Karl Marx, Euvres, Paris, Gallimard, 1963 [1848].

MaLaren David J., David Dale of New Lanark: a Bright Luminary to Scotland, Milngavie, Heatherbank Press, 1983.

-, " David Dale, Robert Owen and the New Lanark schools in the Scottish educational context, 1785-1824 », Conference paper, Hawaii International Conference on Social Sciences, 12-15 juin 2003.

Owen Robert, The Life of Robert Owen written by himself, John Butt (éd.), Londres, Charles Knight, 1971 [1857].

-, A New View of Society and Other Writings, Gregory Claeys (éd.), Londres, Penguin Classics, 1991.

-, Supplementary appendix to the first volume of "The Life of Robert Owen", containing a series of reports, addresses, memorials, and other documents, referred to in that volume, 1803-1820, Londres, Effingham Wilson, 1858.

-, Threading My Way: Twenty-Seven Years of Autobiography, Londres, Trübner \& Co., 1874.

Parry M. L. et Slater T. R. (éds.), The Making of the Scottish Countryside, Londres, Taylor \& Francis, 1980.

POLANYI Karl, La grande transformation : aux origines politiques et économiques de notre temps, Paris, Gallimard, 1944.

SINCLAIR John (éd.), The Statistical Accounts of Scotland, 1791-1799, URL : $<$ http://edina.ac.uk/stat-acc-scot/>.

Smout T. C., «The Landowner and the Planned Village », dans N. T. Phillipson et Rosalind Mitchinson (éds.), Scotland in the Age of Improvement, Edimbourg, Edinburgh University Press, 1970.

—, A History of the Scottish People, 1560-1830, Londres, Fontana Press, 1969.

Whatley Christopher, Scottish Society 1700-1830: Beyond Jacobitism, towards Industrialisation, Manchester, Manchester University Press, 2000. 